This item was submitted to Loughborough's Research Repository by the author.

Items in Figshare are protected by copyright, with all rights reserved, unless otherwise indicated.

\title{
Lateral control of an autonomous vehicle
}

PLEASE CITE THE PUBLISHED VERSION

https://doi.org/10.1109/tiv.2018.2804173

PUBLISHER

(C) Institute of Electrical and Electronics Engineers (IEEE)

VERSION

AM (Accepted Manuscript)

LICENCE

CC BY-NC-ND 4.0

REPOSITORY RECORD

Jiang, Jingjing, and Alessandro Astolfi. 2019. "Lateral Control of an Autonomous Vehicle”. figshare. https://hdl.handle.net/2134/36750. 


\title{
Lateral Control of an Autonomous Vehicle
}

\author{
Jingjing Jiang $^{1}$ and Alessandro Astolfi ${ }^{2}$
}

\begin{abstract}
The asymptotic stabilization problem for a class of nonlinear under-actuated systems is studied and solved. Its solution, together with the back-stepping and the forwarding control design methods, is exploited in the control of the nonlinear lateral dynamics of a vehicle. Even though the theoretical studies of the lateral control of autonomous vehicles are traditionally applied to lane keeping cases, the results can be applied to broader range of areas, such as lane changing cases. The comparison between the performances of the closed-loop systems with the given controller and a typical human driver is given and demonstrates the speediness and the effectiveness of the feedback controller.
\end{abstract}

\section{NOMENCLATURE}

$\beta \quad$ ratio of the lateral speed and the longitudinal speed

$\dot{\psi} \quad$ yaw rate $[\mathrm{rad} / \mathrm{s}]$

$\psi_{L} \quad$ heading error, relative yaw angle [rad]

$\delta \quad$ actual steering angle [rad]

$\delta_{d} \quad$ steering angle at the column system [rad]

$\rho \quad$ road curvature $\left[\mathrm{m}^{-1}\right]$

$\eta_{t} \quad$ width of the tyre contact patch [m]

$a_{y} \quad$ lateral acceleration of the car $\left[\mathrm{m} \times \mathrm{s}^{-2}\right]$

$B_{u} \quad$ damping coefficient of the steering system $[\mathrm{Nm} /(\operatorname{rad} \times \mathrm{s})]$

$C_{f}\left(C_{r}\right)$ front (rear) tyre cornering stiffness [N/rad]

$F_{f}\left(F_{r}\right)$ front (rear) tyre lateral force [N]

$I_{z} \quad$ moment inertia of the car about the yaw-axis $\left[\mathrm{kg} \times \mathrm{m}^{2}\right]$

$J_{s} \quad$ moment of inertia of the steering system $\left[\mathrm{kg} \times \mathrm{m}^{2}\right]$

$K_{a} \quad$ visual anticipatory control of the driver

$K_{c} \quad$ proportional gain of the transfer function representing the compensatory steering control of the driver

$l_{f}\left(l_{r}\right) \quad$ distances of the front (rear) tyres to the mass center $[\mathrm{m}]$

$m \quad$ mass of the car $[\mathrm{kg}]$

$R_{s} \quad$ reduction ratio of the steering system, i.e. $R_{s}=\delta_{d} / \delta$

$T_{c} \quad$ torque generated by the controller $[\mathrm{Nm}]$

$T_{i}\left(T_{l}\right) \quad$ lag (lead) time constant of the transfer function representing the compensatory steering control of the driver

$T_{n} \quad$ neuromuscular lag time constant of the driver

$T_{p} \quad$ driver's preview time [s]

${ }^{1} \mathrm{~J}$. Jiang is with the Department of Electrical and Electronic Engineering, Imperial College London, UK, E-mail: jingjing.jiang10@imperial.ac.uk

${ }^{2} \mathrm{~A}$. Astolfi is with the Dept. of Electrical and Electronic Engineering, Imperial College London, London, SW7 2AZ, UK and the DICII, University of Roma "Tor Vergata", Via del Politecnico 1, 00133 Rome, Italy, E-mail: a.astolfi@imperial.ac.uk
$T_{s} \quad$ self-aligning moment of the steering system [Nm]

$v_{x} \quad$ longitudinal speed of the car $[\mathrm{m} / \mathrm{s}]$

$v_{y} \quad$ lateral speed of the car $[\mathrm{m} / \mathrm{s}]$

$y_{L} \quad$ lateral deviation of the car $[\mathrm{m}]$

\section{INTRODUCTION}

The study on auto-driving cars is a rapidly growing research area. Many companies across the world, such as Google ${ }^{\circledR}$ and Tesla $^{\circledR}$, are investing millions of dollars in the development of self-driving autonomous cars. Compared with traditional cars controlled completely by the human driver and the cars with passive shared-controller (e.g. anti-skid brake system (ABS)), self-driving cars have the following advantages: the number of car accidents could be reduced drastically and thousands of lives saved; traffic congestions can be significantly reduced and energy efficiency can be greatly improved; people can spend their commuting time on other more valuable things; it is more convenient for the elderly, the children and the disabled to use cars.

To achieve autonomous driving along a pre-defined trajectory, such as a route planned by Google Map ${ }^{\circledR}$ from the house where you live to the place where you work, the control problems for the longitudinal and lateral dynamics of the vehicle have to be studied and solved. Even though the longitudinal dynamics and the lateral dynamics of the vehicle are coupled, it is common to assume that the two are decoupled whenever the road curvature is small [1]. The paper [2] has investigated the control of vehicle longitudinal dynamics, while this paper focuses on the control of the lateral dynamics.

The lateral dynamics of vehicles has been discussed in [3], in which the kinematic and the dynamic models for lateral vehicle motion have been studied. Many control schemes have been established to control the lateral dynamics. The papers [4] and [5] have proposed control laws based on ProportionalIntegral-Derivative (PID) control, while the paper [6] has developed a lateral controller based on fuzzy control. Other control technologies have also been used, such as $H_{\infty}$ control [7], [8], Linear Quadratic Regulator (LQR) [9] and sliding mode control [10], [11], [12]. In addition, the paper [13] has presented a feedback controller to control the lateral dynamics of rear-wheel drive cars subject to state constraints. However, these control methods are developed based on the linearized model of the lateral dynamics, while this paper studies the control problem for the nonlinear lateral dynamics. Model Predictive Control (MPC) is another method used in the trajectory tracking of autonomous cars, for example [14], [15], [16]. The computation time of nonlinear MPC is the main disadvantage of such an approach: this paper proposes an analytical solution to the lateral control problem which does not 
cause any computation burdens. Finally, the papers [17], [18] have studied the lateral control of independently actuated fourwheeled vehicles. Comparisons of different lateral controllers can be found in [19], [20].

The main contributions of the paper are stated as follows. The paper studies the asymptotic stabilization problem for a class of nonlinear systems and exploits the solution to solve the lateral control problem. A Lyapunov-like analysis is used to prove the stability of the closed-loop system.

This paper is organized as follows. Section II describes the model we study in the paper and formulates the lateral control problem for autonomous vehicles. Some preliminary theorems which are essential to design the lateral controller are presented and proved in Section III, while the solution to the lateral control problem is given in Section IV, in which formal properties of the closed-loop system are presented. Section V studies three examples and shows how the controller works in different cases. By comparing the simulation results with typical driver performances we demonstrate the effectiveness of the established controller. Finally, conclusions and suggestions for future work are given in Section VI.

\section{SYSTEM MODELING AND PROBLEM STATEMENT}

Assumption 1: We assume that the longitudinal speed is constant, i.e. $v_{x}$ is a constant, and strictly positive.

Based on the well-known Newton's Second Law for motion along the lateral axis we have

$$
m a_{y}=F_{f}+F_{r},
$$

where $a_{y}$ denotes the lateral acceleration of the car, $F_{f}$ and $F_{r}$ represent the lateral tyre forces of the front and rear wheels, respectively. The lateral acceleration is caused by the motion along the lateral axis (i.e. the $y$-axis) and the centripetal acceleration $v_{x} \dot{\psi}$, where $v_{x}$ and $\dot{\psi}$ are the longitudinal speed of the car and the yaw rate, respectively. Hence, the lateral translational motion can be described as

$$
m\left(\dot{v}_{y}+v_{x} \dot{\psi}\right)=F_{f}+F_{r},
$$

where $m$ is the mass of the car. In addition, the moment balance about the vertical axis yields the equation for the yaw dynamics

$$
I_{z} \ddot{\psi}=F_{f} l_{f}-F_{r} l_{r},
$$

where $I_{z}$ denotes the moment of inertia of the car about the yaw-axis, and $l_{f}$ and $l_{r}$ represent the distances of the front tyre and the rear tyre to the vehicle mass center, respectively.

The side-slip angles for the front and the rear wheels of the car, $\alpha_{f}$ and $\alpha_{r}$, are defined in Fig. 1, where $v$ denotes the velocity of the vehicle, i.e. $v$ is the combination of the longitudinal speed $v_{x}$ and the lateral speed $v_{y}$, and $\delta$ is the steering angle. In addition, $\theta_{f}$ and $\theta_{r}$ represent the angles between the velocity

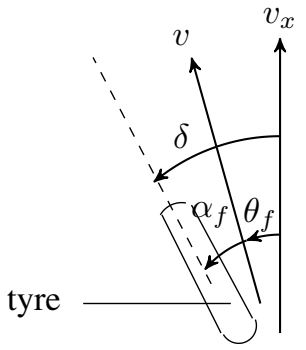

(a) Front Tyre Side-Slip Angle

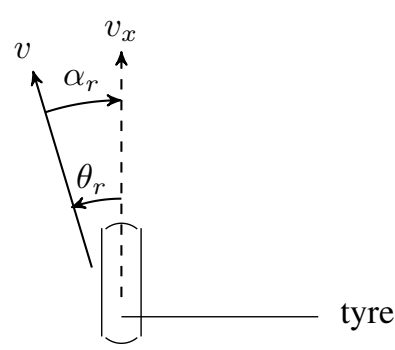

(b) Rear Tyre Side-Slip Angle
Fig. 1: Definitions of Tyre Side-Slip Angles

vector $v$ and the longitudinal speed direction, respectively, and can be calculated as

$$
\tan \left(\theta_{f}\right)=\frac{v_{y}+l_{f} \dot{\psi}}{v_{x}}, \quad \tan \left(\theta_{r}\right)=\frac{v_{y}-l_{r} \dot{\psi}}{v_{x}} .
$$

As detailed in [3], the lateral tyre forces are proportional to the side-slip angle if the angles are small. Therefore, the lateral forces are calculated as

$$
F_{f}=2 C_{f}\left(\delta-\theta_{f}\right), \quad F_{r}=2 C_{r}\left(-\theta_{r}\right),
$$

where $C_{f}$ and $C_{r}$ are the front and rear tyre cornering stiffness, respectively.

We define a new variable $\beta$ as

$$
\beta=\frac{v_{y}}{v_{x}},
$$

which describes the ratio between the lateral velocity and the longitudinal velocity. Using the new variable $\beta$ and substituting (3) and (4) into the equations (1)-(2), the lateral dynamics of the vehicle can be rewritten as

$$
\begin{aligned}
\dot{\beta}= & \frac{2 C_{f}}{m v_{x}} \delta-\dot{\psi}-\frac{2 C_{f}}{m v_{x}} \arctan \left(\beta+\frac{l_{f} \dot{\psi}}{v_{x}}\right) \\
& -\frac{2 C_{r}}{m v_{x}} \arctan \left(\beta-\frac{l_{r} \dot{\psi}}{v_{x}}\right), \\
\ddot{\psi}= & \frac{2 C_{f} l_{f}}{I_{z}} \delta-\frac{2 C_{f} l_{f}}{I_{z}} \arctan \left(\beta+\frac{l_{f} \dot{\psi}}{v_{x}}\right) \\
& +\frac{2 C_{r} l_{r}}{I_{z}} \arctan \left(\beta-\frac{l_{f} \dot{\psi}}{v_{x}}\right),
\end{aligned}
$$

where the steering angle $\delta$ is the "input" signal.

Note that the steering angle cannot be controlled directly in a car. Instead, it is controlled by the steering torque. The dynamics of the steering system is given by the equation

$$
J_{s} \ddot{\delta}_{d}+B_{u} \dot{\delta}_{d}=T_{c}-T_{s},
$$

where $\delta_{d}=\delta R_{s}, R_{s}$ is the reduction ratio of the steering system, and $J_{s}$ and $B_{u}$ represent the moment of inertia and the damping coefficient of the steering system, respectively. $T_{c}$ is the control input, while $T_{s}$ is the self-aligning moment and is calculated as

$$
T_{s}=-\frac{2 C_{f} \eta_{t}}{R_{s}} \beta-\frac{2 C_{f} l_{f} \eta_{t}}{R_{s} v_{x}} \dot{\psi}+\frac{2 C_{f} \eta_{t}}{R_{s}^{2}} \delta_{d} .
$$




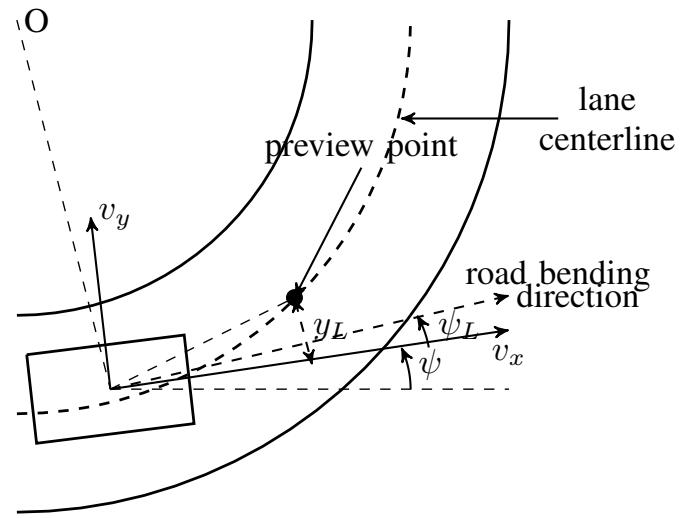

Fig. 2: Graphical Definitions of Variables $y_{L}$ and $\psi_{L}$ in Lane Keeping Cases.

In trajectory tracking (lane keeping) cases, two additional variables are used to describe the relationship between the vehicle and the reference trajectory (central line of the lane). They are the lateral deviation $y_{L}$ and the heading error $\psi_{L}$. Their dynamics can be described by the equations

$$
\begin{aligned}
& \dot{y}_{L}=v_{x} \beta+T_{p} v_{x} \dot{\psi}+v_{x} \psi_{L}, \\
& \dot{\psi}_{L}=\dot{\psi}-v_{x} \rho,
\end{aligned}
$$

where $\rho$ denotes the curvature of the reference trajectory (lane curvature). Note that the graphical definitions of the variables are illustrated in Fig. 2.

Definition 1: The signal $\rho(t)$ is said to be "feasible" if and only if there exist functions $\beta(t), \dot{\psi}(t), \delta(t), \dot{\delta}(t), y_{L}(t), \psi_{L}(t)$ and $T_{c}(t)$ such that the equations (5)-(6)-(7)-(8) hold for all $t \geq 0$.

Suppose $\rho(t)$ is feasible and $v_{x}$ is a given positive constant. Then the references for the variables $\beta(t), \dot{\psi}(t), \delta(t), y_{L}(t), \psi_{L}(t), T_{c}(t)$ satisfy the equations

$$
\begin{aligned}
\dot{\beta}_{r}= & \frac{2 C_{f}}{m v_{x}} \delta_{r}-\dot{\psi}_{r}-\frac{2 C_{f}}{m v_{x}} \arctan \left(\beta_{r}+\frac{l_{f} \dot{\psi}_{r}}{v_{x}}\right) \\
& -\frac{2 C_{r}}{m v_{x}} \arctan \left(\beta-\frac{l_{r} \dot{\psi}}{v_{x}}\right), \\
\ddot{\psi}_{r}= & \frac{2 C_{f} l_{f}}{I_{z}} \delta_{r}-\frac{2 C_{f} l_{f}}{I_{z}} \arctan \left(\beta_{r}+\frac{l_{f} \dot{\psi}_{r}}{v_{x}}\right) \\
& +\frac{2 C_{r} l_{r}}{I_{z}} \arctan \left(\beta_{r}-\frac{l_{f} \dot{\psi}_{r}}{v_{x}}\right), \\
\ddot{\delta}_{r}= & \frac{T_{c_{r}}-T_{s}-B_{u} R_{s} \dot{\delta}_{r}}{J_{s} R_{s}}, \\
\dot{y}_{L_{r}}= & v_{x} \beta_{r}+T_{p} v_{x} \dot{\psi}_{r}+v_{x} \psi_{L_{r}}, \\
\dot{\psi}_{L_{r}}= & \dot{\psi}_{r}-v_{x} \rho,
\end{aligned}
$$

where

$$
T_{s}=-\frac{2 C_{f} \eta_{t}}{R_{s}} \beta_{r}-\frac{2 C_{f} l_{f} \eta_{t}}{R_{s} v_{x}} \dot{\psi}_{r}+\frac{2 C_{f} \eta_{t}}{R_{s}} \delta_{r} .
$$

The control problem for the lateral motion can then be formulated as follows.
Given the system (5)-(6)-(7)-(8) and the time history of the feasible road curvature $\rho(t)$, find (if possible) a feedback controller $T_{c}(t)$ such that the closed-loop system has the following properties.

P1) The control effort is bounded, i.e. $\exists \mathcal{B}>0$ such that $\left|T_{c}(t)\right| \leq \mathcal{B}$ for all $t \geq 0$.

P2) The system state converges to its reference value, i.e.

$$
\begin{aligned}
\lim _{t \rightarrow \infty}\left(\beta(t), \dot{\psi}(t), \delta(t), y_{L}(t), \psi_{L}(t)\right)- \\
\quad\left(\beta_{r}(t), \dot{\psi}_{r}(t), \delta_{r}(t), y_{L_{r}}(t), \psi_{L_{r}}(t)\right)=0,
\end{aligned}
$$

where $\beta_{r}, \dot{\psi}_{r}, \delta_{r}, y_{L_{r}}$ and $\psi_{L_{r}}$ are feasible reference signals.

P3) $\lim _{t \rightarrow \infty} T_{c}(t)-T_{c_{r}}(t)=0$, where $T_{c_{r}}$ is defined in (9).

\section{PRELIMINARY THEOREMS}

This section provides two basic results used to design the controller for the under-actuated nonlinear system (5).

Theorem 1: Consider a two dimensional system, the dynamics of which can be described by the equations

$$
\begin{aligned}
& \dot{s}_{1}=q_{11} s_{1}+p_{1}\left(s_{2}\right), \\
& \dot{s}_{2}=p_{2}\left(s_{1}, s_{2}\right)+g u,
\end{aligned}
$$

where $s_{1}$ and $s_{2}$ are the states of the system, $u$ is the control input, and $p_{1}: \mathbb{R} \rightarrow \mathbb{R}$ and $p_{2}: \mathbb{R}^{2} \rightarrow \mathbb{R}$ denote two nonlinear mappings. Assume that

A1) $g \neq 0$;

A2) $q_{11}<0$

A3) The function $\frac{p_{1}\left(s_{2}\right)}{s_{2}}$ is continuous at $s_{2}=0$.

Then there exists a state-feedback controller $u\left(s_{1}, s_{2}\right)$ such that the zero equilibrium of the closed-loop-system is globally asymptotically stable. One such a choice is given by

$$
u\left(s_{1}, s_{2}\right)=-\frac{k s_{2}+s_{1} \frac{p_{1}\left(s_{2}\right)}{s_{2}}+p_{2}\left(s_{1}, s_{2}\right)}{g},
$$

for any $k>0$.

Proof: Consider the Lyapunov function

$$
L=\frac{1}{2}\left(s_{1}^{2}+s_{2}^{2}\right)
$$

Its time derivative along the trajectories of the closed-loop system is

$$
\dot{L}=s_{1}\left[q_{11} s_{1}+p_{1}\left(s_{2}\right)\right]+s_{2}\left[p_{2}\left(s_{1}, s_{2}\right)+g u\right] .
$$

By A3),

$$
\dot{L}=q_{11} s_{1}^{2}+s_{2}\left[s_{1} \frac{p_{1}\left(s_{2}\right)}{s_{2}}+p_{2}\left(s_{1}, s_{2}\right)+g u\right] .
$$


Substituting (11) into the above equation yields

$$
\dot{L}=q_{11} s_{1}^{2}-k s_{2}^{2} .
$$

By A2) and the fact that $k>0$ we conclude that

$$
\dot{L}<0
$$

for all $\left(s_{1}, s_{2}\right) \neq(0,0)$. Furthermore,

$$
\dot{L}=0 \Longleftrightarrow s_{1}=s_{2}=0 .
$$

Therefore, the claim holds.

Theorem 2: Consider a system with two degrees-of-freedom, the dynamics of which is described by the equations

$$
\begin{aligned}
& \dot{s}_{1}=a_{11} s_{1}+a_{12} s_{2}+f_{1}\left(s_{2}\right)+b_{1} u, \\
& \dot{s}_{2}=a_{21} s_{1}+a_{22} s_{2}+f_{2}\left(s_{2}\right)+b_{2} u,
\end{aligned}
$$

with $s_{1}(t) \in \mathbb{R}, s_{2}(t) \in \mathbb{R}$ and $u(t) \in \mathbb{R}$. $a_{11}, a_{12}, a_{21}, a_{22}, b_{1}$ and $b_{2}$ are constant parameters, while $f_{1}\left(s_{2}\right)$ and $f_{2}\left(s_{2}\right)$ are continuous functions of $s_{2}$. Suppose that

H1) $\frac{f_{1}\left(s_{2}\right)}{s_{2}}$ and $\frac{f_{2}\left(s_{2}\right)}{s_{2}}$ are continuous at $s_{2}=0$;

H2) $b_{2}\left(b_{1} a_{21}-b_{2} a_{11}\right)>0$.

Then there exists a state-feedback controller $u\left(s_{1}, s_{2}\right)$ such that the zero equilibrium of the closed-loop system is globally asymptotically stable. One such a choice is given by the feedback

$$
\begin{aligned}
u\left(s_{1}, s_{2}\right)= & \frac{-a_{21} s_{1}-a_{22} s_{2}-f_{2}\left(s_{2}\right)+b_{1}\left(b_{1} a_{21}-b_{2} a_{11}\right) s_{1}}{b_{2}} \\
& -\frac{\left(b_{1} s_{2}-b_{2} s_{1}\right)\left[b_{1} a_{22}-b_{2} a_{12}+b_{1} \frac{f_{2}\left(s_{2}\right)}{s_{2}}\right]}{b_{2}} \\
& -\frac{-\left(b_{1} s_{2}-b_{2} s_{1}\right) b_{2} \frac{f_{1}\left(s_{2}\right)}{s_{2}}+k s_{2}}{b_{2}},
\end{aligned}
$$

for any $k>0$.

\section{Proof: Define}

$$
\tilde{s}_{1} \triangleq\left(b_{1} s_{2}-b_{2} s_{1}\right)
$$

and note that

$$
\begin{aligned}
\dot{\tilde{s}}_{1}= & \left(b_{1} a_{21}-b_{2} a_{11}\right) s_{1}+\left(b_{1} a_{22}-b_{2} a_{12}\right) s_{2} \\
& +\left[b_{1} f_{2}\left(s_{2}\right)-b_{2} f_{1}\left(s_{2}\right)\right] \\
= & s_{2}\left[b_{1} a_{22}-b_{2} a_{12}+b_{1} \frac{f_{2}\left(s_{2}\right)}{s_{2}}-b_{2} \frac{f_{1}\left(s_{2}\right)}{s_{2}}\right] \\
& +\left(b_{1} a_{21}-b_{2} a_{11}\right) s_{1},
\end{aligned}
$$

hence

$$
\begin{aligned}
& \dot{\tilde{s}}_{1}=q_{11} \tilde{s}_{1}+p_{1}\left(s_{2}\right), \\
& \dot{s}_{2}=p_{2}\left(\tilde{s}_{1}, s_{2}\right)+b_{2} u,
\end{aligned}
$$

where

$$
\begin{gathered}
q_{11}=a_{11}-\frac{b_{1}}{b_{2}} a_{21}, \\
p_{1}\left(s_{2}\right)=s_{2}\left[\begin{array}{c}
b_{1} a_{22}-b_{2} a_{12}+b_{1} \frac{f_{2}\left(s_{2}\right)}{s_{2}}-b_{2} \frac{f_{1}\left(s_{2}\right)}{s_{2}} \\
+\frac{b_{1}}{b_{2}}\left(b_{1} a_{21}-b_{2} a_{11}\right)
\end{array}\right], \\
p_{2}\left(\tilde{s}_{1}, s_{2}\right)=a_{21} \frac{b_{1} s_{2}-\tilde{s}_{1}}{b_{2}}+a_{22} s_{2}+f_{2}\left(s_{2}\right) .
\end{gathered}
$$

We now exploit Theorem 1, for which we need to check if A1) to A3) hold. To this end, note that

$$
\mathrm{H} 2) \Longrightarrow \frac{b_{2}\left(b_{1} a_{21}-b_{2} a_{11}\right)}{b_{2}^{2}}>0 \Longrightarrow \frac{b_{1}}{b_{2}} a_{21}-a_{11}>0 .
$$

Therefore, $q_{11}<0$, i.e. A2) in Theorem 1 holds. Moreover, A1) and A3) are direct consequences of $\mathrm{H} 2$ ) and $\mathrm{H} 1$ ), respectively.

According to Theorem 1, the state-feedback control law

$$
u\left(\tilde{s}_{1}, s_{2}\right)=-\frac{k s_{2}+\tilde{s}_{1} \frac{p_{1}\left(s_{2}\right)}{s_{2}}+p_{2}\left(\tilde{s}_{1}, s_{2}\right)}{b_{2}},
$$

with $k>0$, globally asymptotically stabilizes the zero equilibrium of system (12).

Rewriting the equation (14) with the variable $s_{1}$ and $s_{2}$ yields

$$
\begin{aligned}
u\left(s_{1}, s_{2}\right)= & -\frac{k s_{2}+a_{21} \frac{b_{1} s_{2}-b_{1} s_{2}+b_{2} s_{1}}{b_{2}}+a_{22} s_{2}+f_{2}\left(s_{2}\right)}{b_{2}} \\
& -\frac{\left(b_{1} s_{2}-b_{2} s_{1}\right)\left(b_{1} a_{22}-b_{2} a_{12}+b_{1} \frac{f_{2}\left(s_{2}\right)}{s_{2}}\right)}{b_{2}} \\
& +\frac{\left(b_{1} s_{2}-b_{2} s_{1}\right)\left[b_{2} \frac{f_{1}\left(s_{2}\right)}{s_{2}}-\frac{b_{1}}{b_{2}}\left(b_{1} a_{21}-b_{2} a_{11}\right)\right]}{b_{2}},
\end{aligned}
$$

with $k>0$, that is the control law (13).

\section{LATERAL CONTROL DESIGN FOR AUTONOMOUS VEHICLES}

To design the lateral controller we regard the overall system as an inter connected system as depicted in Fig. 3. It is clear that the open-loop system contains three components, i.e. the 'Lateral Dynamics of the Vehicle', the 'Lane Keeping Dynamics' and the 'Steering System' together with the 'SelfAligning Moment', of which the core subsystem is the "Lateral Dynamics of the Vehicle". This section gives a solution to the control problem stated in Section II. 


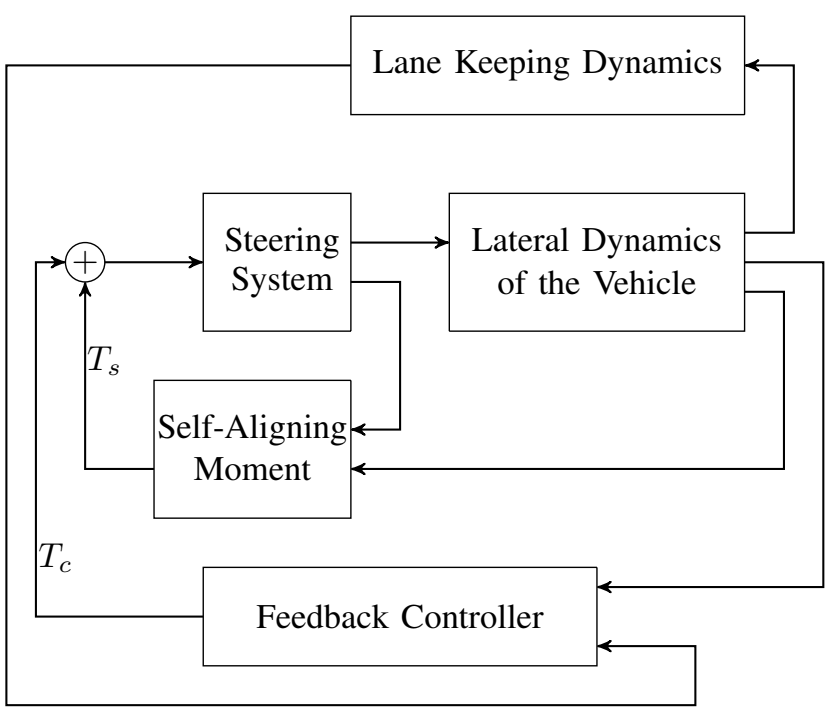

Fig. 3: Block Diagram of the System

\section{A. Control Design for the Lateral Dynamics of the Vehicle}

Consider the subsystem named as 'Lateral Dynamics of the Vehicle' in Fig. 3. This subsection studies how to design a controller for the nonlinear system (5) based on the preliminary results presented in Section III.

Define the variables $x_{1}$ and $x_{2}$ as

$$
x_{1}=\beta+\frac{l_{f}}{v_{x}} \dot{\psi}, \quad x_{2}=\beta-\frac{l_{r}}{v_{x}} \dot{\psi} .
$$

With the new variables the system (5) can then be rewritten as

$$
\begin{aligned}
\dot{x}_{1}= & -\frac{v_{x}}{l_{f}+l_{r}} x_{1}+\frac{v_{x}}{l_{f}+l_{r}} x_{2}+\left(\frac{2 C_{r} l_{r} l_{f}}{I_{z} v_{x}}-\frac{2 C_{r}}{m v_{x}}\right) \arctan x_{2} \\
& +\left(\frac{2 C_{f} l_{f}^{2}}{I_{z} v_{x}}+\frac{2 C_{f}}{m v_{x}}\right)\left(\delta-\arctan x_{1}\right), \\
\dot{x}_{2}= & -\frac{v_{x}}{l_{f}+l_{r}} x_{1}+\frac{v_{x}}{l_{f}+l_{r}} x_{2}-\left(\frac{2 C_{r} l_{r}^{2}}{I_{z} v_{x}}+\frac{2 C_{r}}{m v_{x}}\right) \arctan x_{2} \\
& +\left(\frac{2 C_{f}}{m v_{x}}-\frac{2 C_{f} l_{f} l_{r}}{I_{z} v_{x}}\right)\left(\delta-\arctan x_{1}\right) .
\end{aligned}
$$

Regarding $\left(\delta-\arctan x_{1}\right)$ as an auxiliary input signal $\tilde{\delta}$, the system (16) can be rewritten in the form studied in Theorem 2 as

$$
\begin{aligned}
& \dot{x}_{1}=a_{11} x_{1}+a_{12} x_{2}+f_{1}\left(x_{2}\right)+b_{1} \tilde{\delta}, \\
& \dot{x}_{2}=a_{21} x_{1}+a_{22} x_{2}+f_{2}\left(x_{2}\right)+b_{2} \tilde{\delta},
\end{aligned}
$$

where

$$
\begin{array}{r}
a_{11}=a_{21}=-a_{12}=-a_{22}=-\frac{v_{x}}{l_{f}+l_{r}}, \\
b_{1}=\frac{2 C_{f} l_{f}^{2}}{I_{z} v_{x}}+\frac{2 C_{f}}{m v_{x}}, \quad b_{2}=\frac{2 C_{f}}{m v_{x}}-\frac{2 C_{f} l_{f} l_{r}}{I_{z} v_{x}},
\end{array}
$$

$$
\begin{array}{r}
f_{1}\left(x_{2}\right)=\left(\frac{2 C_{r} l_{r} l_{f}}{I_{z} v_{x}}-\frac{2 C_{r}}{m v_{x}}\right) \arctan x_{2}, \\
f_{2}\left(x_{2}\right)=\left(\frac{2 C_{r} l_{r}^{2}}{I_{z} v_{x}}+\frac{2 C_{r}}{m v_{x}}\right) \arctan x_{2} .
\end{array}
$$

Note that $b_{1}>0$ and $a_{11}=a_{21}<0$.

Assumption 2: For typical values of car parameters $b_{2}<0$.

By Assumption 2 and the previous analysis on the signs of the parameters $a_{11}, a_{21}$ and $b_{1}$ in (17), H2) in Theorem 2 holds. In addition, the function $\frac{\arctan x}{x}$ is continuous at $x=0$, indicating that H1) of Theorem 2 holds.

According to Theorem 2, there exists a state-feedback controller $\tilde{\delta}\left(x_{1}, x_{2}\right)$ such that the zero equilibrium of the closedloop system is globally asymptotically stable. One choice of $\tilde{\delta}\left(x_{1}, x_{2}\right)$ is given by

$$
\begin{aligned}
\tilde{\delta}= & \frac{-a_{21} x_{1}-a_{22} x_{2}-f_{2}\left(x_{2}\right)+b_{1}\left(b_{1} a_{21}-b_{2} a_{11}\right) x_{1}}{b_{2}} \\
& -\frac{\left(b_{1} x_{2}-b_{2} x_{1}\right)\left[b_{1} a_{22}-b_{2} a_{12}+b_{1} \frac{f_{2}\left(x_{2}\right)}{x_{2}}\right]}{b_{2}} \\
& -\frac{-\left(b_{1} x_{2}-b_{2} x_{1}\right) b_{2} \frac{f_{1}\left(x_{2}\right)}{x_{2}}+k_{1} x_{2}}{b_{2}},
\end{aligned}
$$

where $a_{11}, a_{12}, a_{21}, a_{22}, b_{1}, b_{2}, f_{1}\left(x_{2}\right)$ and $f_{2}\left(x_{2}\right)$ are defined in (17) and $k_{1}>0$.

Lemma 1: Consider the system (5) with the feedback controller $\delta=\tilde{\delta}+\arctan x_{1}$, where $\tilde{\delta}$ is given by (18) and $x_{1}$ and $x_{2}$ are defined in (15). The origin is a globally asymptotically stable equilibrium.

Proof: It is a direct consequence of Theorem 2.

\section{B. Control Design for the Overall System}

In this subsection we provide a control design for the overall system (5)-(6)-(7)-(8) in the case in which $\rho(t)=0$ for all $t \geq 0$. In such a case $\left(\beta, \psi, \delta, y_{L}, \psi_{L}\right)=(0,0,0,0,0)$ is an equilibrium of the system (5)-(6)-(7)-(8), hence we define the reference trajectory as

$$
\beta_{r}(t)=\dot{\psi}_{r}(t)=\delta_{r}(t)=\dot{\delta}_{r}(t)=y_{L_{r}}(t)=\psi_{L_{r}}(t)=0,
$$

for all $t \geq 0$.

Definition 2: Let $\phi(x): \mathbb{R} \rightarrow \mathbb{R}$ be defined as

$$
\phi(x)= \begin{cases}1, & \text { if } x>\sqrt{2}, \\ \sqrt{1-(\sqrt{2}-x)^{2}}, & \text { if } \frac{\sqrt{2}}{2}<x \leq \sqrt{2}, \\ x, & \text { if }-\frac{\sqrt{2}}{2} \leq x \leq \frac{\sqrt{2}}{2}, \\ -\sqrt{1-(\sqrt{2}-x)^{2},}, & \text { if }-\sqrt{2} \leq x<-\frac{\sqrt{2}}{2}, \\ -1, & \text { if } x<-\sqrt{2} .\end{cases}
$$




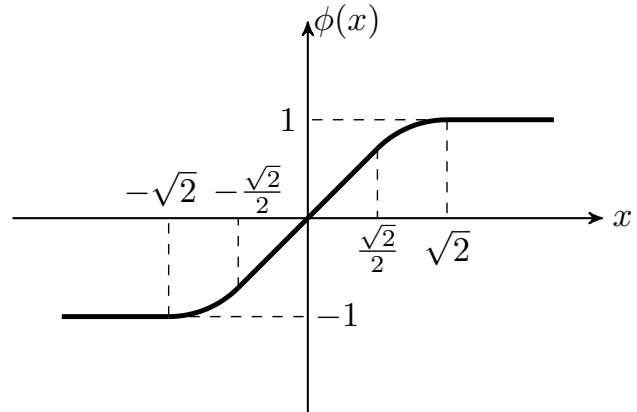

Fig. 4: The graph of the function $\phi(x)$ in Definition 2.

Fig. 4 illustrates the graph of the function $\phi(x)$. Note that $\phi(x)$ is twice differentiable.

On the basis of the control design for feedforward systems proposed in [21], we can derive the following result.

Lemma 2: Consider the lateral dynamics of the vehicle (5) together with the lane keeping dynamics (8). There exists $\kappa_{1}^{*}>0, \kappa_{2}^{*}>0, \epsilon_{1}^{*}>0$ and $\epsilon_{2}^{*}>0$ such that for any $\kappa_{1} \in\left(0, \kappa_{1}^{*}\right), \kappa_{2} \in\left(0, \kappa_{2}^{*}\right), \epsilon_{1} \in\left(0, \epsilon_{1}^{*}\right)$ and $\epsilon_{2} \in\left(0, \epsilon_{2}^{*}\right)$ the zero equilibrium of the closed-loop system (5)-(8) with the controller

$$
\delta=\delta^{*}=\tilde{\delta}+\arctan x_{1}-\epsilon_{1} \phi\left(\frac{\kappa_{1}}{\epsilon_{1}} \psi_{L}\right)-\epsilon_{2} \phi\left(\frac{\kappa_{2}}{\epsilon_{2}} y_{L}\right)
$$

where $\tilde{\delta}$ and $x_{1}$ are defined in (18) and (15), respectively, is globally asymptotically stable.

Proof: In the case $\rho=0$, the lane keeping dynamics described by the equations (8) are functions of the variables $\beta$ and $\dot{\psi}$. Based on the relationship between the variables $\left(x_{1}, x_{2}\right)$ and the variables $(\beta, \dot{\psi})$ given in (15), the system (8) can be rewritten as

$$
\begin{aligned}
\dot{y}_{L}= & v_{x}\left(\frac{l_{r}}{l_{f}+l_{r}} x_{1}+\frac{l_{f}}{l_{f}+l_{r}} x_{2}\right)+v_{x} \psi_{L}, \\
& +T_{p} v_{x}\left(\frac{v_{x}}{l_{f}+l_{r}} x_{1}-\frac{v_{x}}{l_{f}+l_{r}} x_{2}\right) \\
\dot{\psi}_{L}= & \frac{v_{x}}{l_{f}+l_{r}} x_{1}-\frac{v_{x}}{l_{f}+l_{r}} x_{2},
\end{aligned}
$$

The linear approximation of the system (16) at the zero equilibrium point can be written as

$$
\dot{x}=F x+\left[\begin{array}{l}
b_{1} \\
b_{2}
\end{array}\right] \tilde{\delta},
$$

where

$$
F=\left[\begin{array}{cc}
a_{11} & a_{12}+\frac{2 C_{r} l_{r} l_{f}}{I_{z} v_{x}}-\frac{2 C_{r}}{m v_{x}} \\
a_{21} & a_{22}+\frac{2 C_{r} l_{r}^{2}}{I_{z} v_{x}}+\frac{2 C_{r}}{m v_{x}}
\end{array}\right]
$$

Since the zero equilibrium of the system (16) with the control law (18) is globally asymptotically stable, the zero equilibrium of the closed-loop system (16)-(19)-(20) is globally asymptotically stable by applying Proposition 1 in [21] twice, hence the claim.

Note that the signal $\delta^{*}$ calculated in (19) is smooth and twice differentiable.

Finally, based on the back-stepping technique in [22], we obtain a control law for the overall system.

Theorem 3: Let $\rho(t)=0$. Consider the system (5)-(6)-(7)-(8) with the controller

$$
\begin{aligned}
T_{c}= & T_{s}+J_{s} R_{s}\left[\ddot{\delta}^{*}-k_{2}\left(\dot{\delta}-\dot{\delta}^{*}\right)\right]+B_{u} R_{s} \dot{\delta} \\
& -k_{3}\left[\dot{\delta}-\dot{\delta}^{*}+k_{2}\left(\dot{\delta}-\dot{\delta}^{*}\right)\right] \\
= & -\frac{2 C_{f} \eta_{t}}{R_{s}} \beta-\frac{2 C_{f} l_{f} \eta_{t}}{R_{s} v_{x}} \dot{\psi}+\frac{2 C_{f} \eta_{t}}{R_{s}} \delta+B_{u} R_{s} \dot{\delta} \\
& +J_{s} R_{s}\left[\ddot{\delta}^{*}-k_{2}\left(\dot{\delta}-\dot{\delta}^{*}\right)\right]-k_{3}\left[\dot{\delta}-\dot{\delta}^{*}+k_{2}\left(\dot{\delta}-\dot{\delta}^{*}\right)\right],
\end{aligned}
$$

where $\delta^{*}$ is calculated in (19). Then the zero equilibrium of the closed-loop system is globally asymptotically stable.

Proof: The overall system can be written as

$$
\begin{aligned}
& \dot{s}=f_{s}(s)+g_{s}(s) \delta, \\
& \ddot{\delta}=\frac{T_{c}-T_{s}}{R_{s} J_{s}}-\frac{B_{u}}{J_{s}} \dot{\delta},
\end{aligned}
$$

where $s=\left[\beta, \dot{\psi}, y_{L}, \psi_{L}\right]^{T}, f_{s}: \mathbb{R}^{4} \rightarrow \mathbb{R}^{4}$ and $g_{s}: \mathbb{R}^{4} \rightarrow \mathbb{R}^{4}$. Lemma 2 holds, hence the zero equilibrium of the subsystem

$$
\dot{s}=f_{s}(s)+g_{s}(s) \delta^{*}(s)
$$

is globally asymptotically stable and the corresponding Lyapunov function is $L(s)$. Using two steps of the back-stepping technique, we can prove that the controller (21) is globally asymptotically stabilizes the zero equilibrium of the system (5)-(6)-(7)-(8), hence the claim.

\section{State-Feedback Tracking Control Design for the Overall System}

This subsection discusses the lateral control design for the overall system (5)-(6)-(7)-(8) with non-zero, but feasible, road curvature $\rho(t)$. We say that $\rho(t)$ is feasible if $\beta_{r}, \dot{\psi}_{r}, \delta_{r}, y_{L_{r}}, \psi_{L_{r}}$ and $T_{c_{r}}$ exist, where $T_{c_{r}}$ denotes the reference control input.

Define the augmented reference signals $x_{1 r}$ and $x_{2 r}$ as

$$
x_{1 r}=\beta_{r}+\frac{l_{f}}{v_{x}} \dot{\psi}_{r}, \quad x_{2 r}=\beta_{r}-\frac{l_{r}}{v_{x}} \dot{\psi}_{r},
$$

and the error signals $x_{1 e}$ and $x_{2 e}$ as

$$
x_{1 e}=x_{1}-x_{1 r}, \quad x_{2 e}=x_{2}-x_{2 r} .
$$

Based on (17) it is easy to derive the equations

$$
\begin{aligned}
& \dot{x}_{1 e}=a_{11} x_{1 e}+a_{12} x_{2 e}+f_{1}\left(x_{2}\right)-f_{1}\left(x_{2 r}\right)+b_{1} \tilde{\delta}_{e}, \\
& \dot{x}_{2 e}=a_{21} x_{1 e}+a_{22} x_{2 e}+f_{2}\left(x_{2}\right)-f_{2}\left(x_{2 r}\right)+b_{2} \tilde{\delta}_{e},
\end{aligned}
$$


where $a_{11}, a_{12}, a_{21}, a_{22}, b_{1}, b_{2}, f_{1}\left(x_{2}\right)$ and $f_{2}\left(x_{2}\right)$ have the same definitions as those in (17). Moreover,

$$
\tilde{\delta}_{e}=\delta-\arctan x_{1}-\delta_{r}+\arctan x_{1 r} .
$$

Assumption 3: Assume that $x_{2}(t) x_{2 r}(t)>-1$ for all $t \geq 0$.

Note that the above assumption holds in typical driving scenarios since the magnitudes of $x_{2}$ and $x_{2 r}$ are small.

Assumption 3 indicates that

$$
\begin{aligned}
\arctan x_{2}-\arctan x_{2 r} & =\arctan \frac{x_{2}-x_{2 r}}{1+x_{2} x_{2 r}} \\
& =\arctan \frac{x_{2 e}}{1+x_{2} x_{2 r}} .
\end{aligned}
$$

Therefore

$$
\begin{aligned}
h_{1}\left(x_{2 e}\right) & \triangleq f_{1}\left(x_{2}\right)-f_{1}\left(x_{2 r}\right) \\
& =\left(\frac{2 C_{r} l_{r} l_{f}}{I_{z} v_{x}}-\frac{2 C_{r}}{m v_{x}}\right) \arctan \frac{x_{2 e}}{1+x_{2} x_{2 r}}, \\
h_{2}\left(x_{2 e}\right) & \triangleq f_{2}\left(x_{2}\right)-f_{2}\left(x_{2 r}\right) \\
& =\left(\frac{2 C_{r} l_{r}^{2}}{I_{z} v_{x}}+\frac{2 C_{r}}{m v_{x}}\right) \arctan \frac{x_{2 e}}{1+x_{2} x_{2 r}} .
\end{aligned}
$$

Hence, the system (22) satisfies all the hypothesis (i.e. H1 and $\mathrm{H} 2$ ) of Theorem 2.

Theorem 4: Consider the system (5)-(6)-(7)-(8) controlled by the feedback controller

$$
\begin{aligned}
T_{c}= & T_{c_{r}}-\frac{2 C_{f} \eta_{t}}{R_{s}} \beta_{e}-\frac{2 C_{f} l_{f} \eta_{t}}{R_{s} v_{x}} \dot{\psi}_{e}+\frac{2 C_{f} \eta_{t}}{R_{s}} \delta_{e}+B_{u} R_{s} \dot{\delta}_{e} \\
& +J_{s} R_{s}\left[\ddot{\tilde{\delta}}_{e}^{*}-k_{2}\left(\dot{\delta}_{e}-\dot{\delta}_{e}^{*}\right)\right]-k_{3}\left[\dot{\delta}_{e}-\dot{\delta}_{e}^{*}+k_{2}\left(\dot{\delta}_{e}-\dot{\delta}_{e}^{*}\right)\right],
\end{aligned}
$$

where

$$
\begin{array}{r}
\delta_{e}^{*}=\tilde{\delta}_{e}+\arctan x_{1 e}-\epsilon_{1} \phi_{e}\left(\frac{\kappa_{1}}{\epsilon_{1}} \psi_{L_{e}}\right)-\epsilon_{2} \phi\left(\frac{\kappa_{2}}{\epsilon_{2}} y_{L_{e}}\right), \\
\delta_{e}=\delta-\delta_{r}, \quad \beta_{e}=\beta-\beta_{r}, \quad \dot{\psi}_{e}=\dot{\psi}-\dot{\psi}_{r}
\end{array}
$$

with

$$
\begin{aligned}
\tilde{\delta}_{e}= & \frac{-a_{21} x_{1 e}-a_{22} x_{2 e}-h_{2}\left(x_{2_{e}}\right)+b_{1}\left(b_{1} a_{21}-b_{2} a_{11}\right) x_{1 e}}{b_{2}} \\
& -\frac{\left(b_{1} x_{2 e}-b_{2} x_{1 e}\right)\left[b_{1} a_{22}-b_{2} a_{12}+b_{1} \frac{h_{2}\left(x_{2 e}\right)}{x_{2 e}}\right]}{b_{2}} \\
& -\frac{-\left(b_{1} x_{2 e}-b_{2} x_{1 e}\right) b_{2} \frac{h_{1}\left(x_{2 e}\right)}{x_{2 e}}+k_{1} x_{2 e}}{b_{2}},
\end{aligned}
$$

$y_{L_{e}}=y_{L}-y_{L_{r}}$ and $\psi_{L_{e}}=\psi_{L}-\psi_{L_{r}} \cdot h_{1}\left(x_{2 e}\right)$ and $h_{2}\left(x_{2 e}\right)$ are defined in (23) and $k_{i}$ for all $i \in\{1,2,3\}$ are positive constants. Suppose that the reference trajectory $\left(\beta_{r}, \dot{\psi}_{r}, \delta_{r}, y_{L_{r}}, \psi_{L_{r}}\right)$ is given and the road curvature $\rho(t)$ is feasible. In addition, we assume that Assumptions 1 to 3 hold. Then there exists $\kappa_{1}^{*}>0, \kappa_{2}^{*}>0, \epsilon_{1}^{*}>0$ and $\epsilon_{2}^{*}>0$ such that for any $\kappa_{1} \in\left(0, \kappa_{1}^{*}\right), \kappa_{2} \in\left(0, \kappa_{2}^{*}\right), \epsilon_{1} \in\left(0, \epsilon_{1}^{*}\right)$ and $\epsilon_{2} \in\left(0, \epsilon_{2}^{*}\right)$ the closed-loop system (5)-(6)-(7)-(8)-(24) has the following properties.

i) The tracking error converges to zero, i.e.

$$
\begin{array}{r}
\lim _{t \rightarrow \infty}\left(y_{L}(t)-y_{L_{r}}(t)\right)=\lim _{t \rightarrow \infty}\left(\psi_{L}(t)-\psi_{L_{r}}(t)\right)=0, \\
\lim _{t \rightarrow \infty}(\beta(t)-\beta r(t))=\lim _{t \rightarrow \infty}\left(\dot{\psi}(t)-\dot{\psi}_{r}(t)\right)=0 .
\end{array}
$$

ii) The control input is bounded, i.e. there exists a positive $\mathcal{T}$ such that $\left|T_{c}(t)\right|<\mathcal{T}$ for all $t \geq 0$.

Proof: According to the previous analysis, the system (22) meets all the hypothesis (i.e. H1 and H2) stated in Theorem 2. Similarly to the proof given in Subsection IV-B, Property i) holds due to Theorem 2, Proposition 1 stated in [21] and the back-stepping method detailed in [22].

Property ii) is a direct consequence of the definition of $T_{c}$ given in (24).

Remark 1: Even though the controller is designed for lane keeping cases, it can also be used to change lanes. In lane changing cases a path planner in the outer loop has to generate a feasible reference trajectory which is then tracked by the car with the established lateral controller (24). Note that the path planning algorithm is not studied in the paper.

\section{SIMULATION RESULTS AND ANALYSIS}

This section discusses three case studies: uniform circular motion, tortuous path tracking and spiral tracking, to demonstrate the effectiveness of the developed control law. The cases are simulated by MATLAB SIMULINK and the results show that the established feedback controller is fast and effective in tracking reference trajectories with time-varying curvature. Note that in all the three cases we assume that the car is driven at a constant forward speed $v_{x}=10 \mathrm{~m} / \mathrm{s}$. In addition, the parameter values for the car in all the simulations are given in Table I.

TABLE I: Vehicle Parameters

\begin{tabular}{||c|c||c|c||c|c||}
\hline$\eta$ & 0.15 & $B_{u}$ & 2.5 & $I_{z}$ & 1500 \\
\hline$C_{f}$ & 170390 & $C_{r}$ & 195940 & $J_{s}$ & 0.05 \\
\hline$l_{f}$ & 1.48 & $l_{r}$ & 1.12 & $m$ & 1625 \\
\hline$R_{s}$ & 12 & & & & \\
\hline
\end{tabular}

\section{A. Driver Model}

To compare the performance of the feedback controller established in the paper with that of typical drivers we use the simplified two-level driver model developed in [23]. The model is a multi-input single-output model based on the observation of a far point and a near point, corresponding to $\theta_{\text {far }}$ and $\theta_{\text {near }}$ in Fig. 5, respectively. The visual far angle $\theta_{\text {far }}$ can be approximately calculated as

$$
\theta_{\text {far }} \approx D \rho .
$$




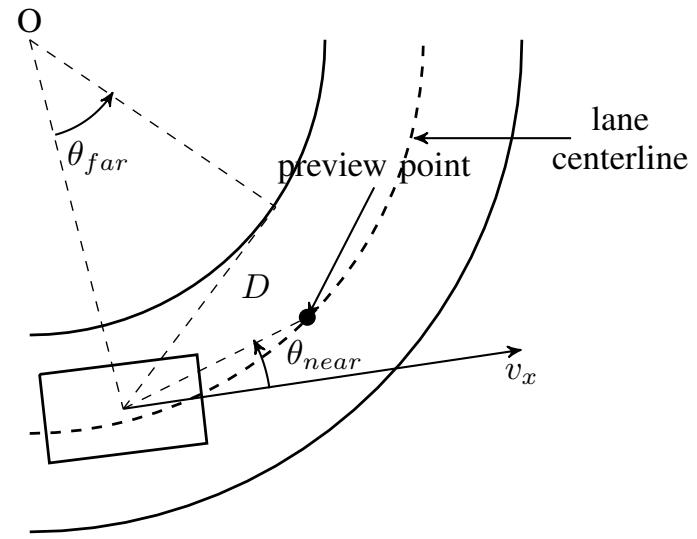

Fig. 5: Visual Angle Definitions in Driving Scenarios.

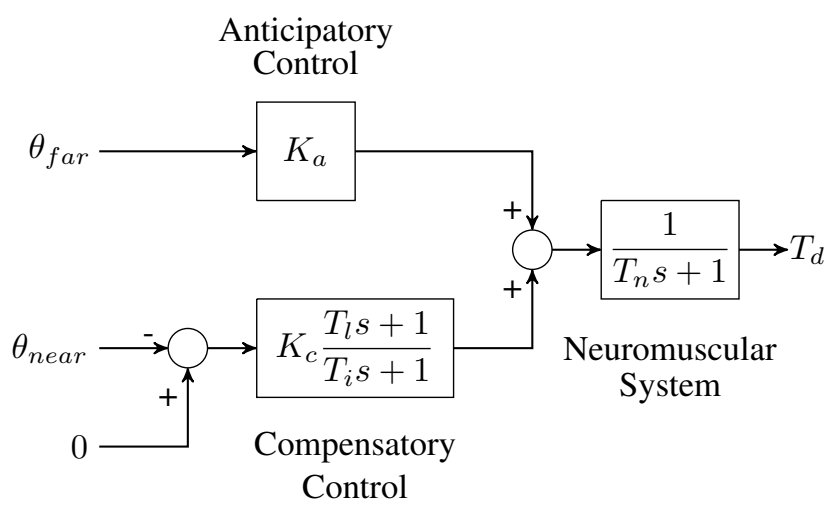

Fig. 6: Simplified Two-Level Driver Model.

In addition, the preview distance is defined as

$$
x_{L}=v_{x} T_{p},
$$

where $T_{p}$ is the driver's preview time. Therefore, the visual near angle $\theta_{\text {near }}$ is calculated as

$$
\theta_{\text {near }}=\frac{y_{L}}{x_{L}}=\frac{y_{L}}{v_{x} T_{p}},
$$

where $y_{L}$ represent the lateral deviation, i.e. one of the states in the system (8).

The block diagram of the driver model is depicted in Fig. 6, where $T_{l}$ and $T_{i}$ denote the lead and lag time constants of the transfer function representing the compensatory steering control of the driver, respectively, while $T_{n}$ represent the constant neuromuscular lag time of the driver. Furthermore, $K_{a}$ and $K_{c}$ describe the anticipatory control of the driver and the proportional gain of the driver with respect to the visual near angular error, respectively. The parameter values of the driver model representing typical drivers are given in Table II, from [23].

TABLE II: Typical Parameter Values for the Driver Model

\begin{tabular}{|c||c||c|c||c||c||c|}
\hline$T_{l}$ & $T_{i}$ & $T_{n}$ & $D$ & $T_{p}$ & $K_{a}$ & $K_{c}$ \\
\hline 1.16 & 0.14 & 0.11 & 15 & 2 & 56.97 & 36.13 \\
\hline
\end{tabular}
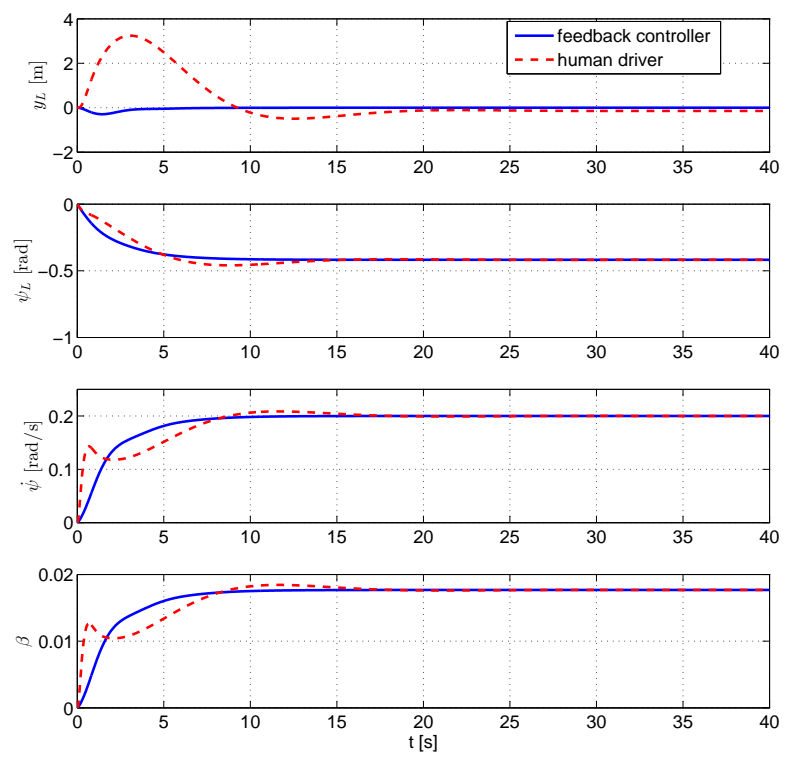

Fig. 7: Uniform Circular Motion. Time histories of the lateral deviation $y_{L}$, heading error $\psi_{L}$, yaw rate $\dot{\psi}$ and the ratio between the lateral speed and the longitudinal speed $\beta$ for the system (5)-(6)-(7)-(8) with the feedback controller (24) and with a typical human driver.

In the following case studies we provide a comparison of simulation results between the performances of the closedloop system with the state-feedback controller and with the typical driver.

\section{B. Uniform Circular Motion}

In this case study we assume that the curvature of the trajectory is constant, i.e. $\rho(t)=0.02$ for all $t \geq 0$. In other words, the reference motion of the car is a uniform circular motion. Note that in the case study we assume that the reference trajectory changes suddenly from a straight line to a circle with radius equal to $50 \mathrm{~m}$. This is different from the case in which the reference path is a straight line connected smoothly with a circle, because the human driver would start turning the steering wheel before entering the circular lane.

Simulation results are displayed in Fig. 7, in which the performance of the feedback controller and the modeled human driver are represented by the blue, solid line and the red, dashed line, respectively. Since all the four key variables related to the lateral dynamics of the vehicle converge to their steady-state values, i.e. reference values, it is obvious that both the feedback controller (24) and the human driver are able to control the system (5)-(6)-(7)-(8). In addition, the car with either of the controllers is able to track the reference path exactly when the curvature of the path is constant. However, by comparing the simulation results for the variable $y_{L}$, it is clear that the response of the closed-loop system with the 

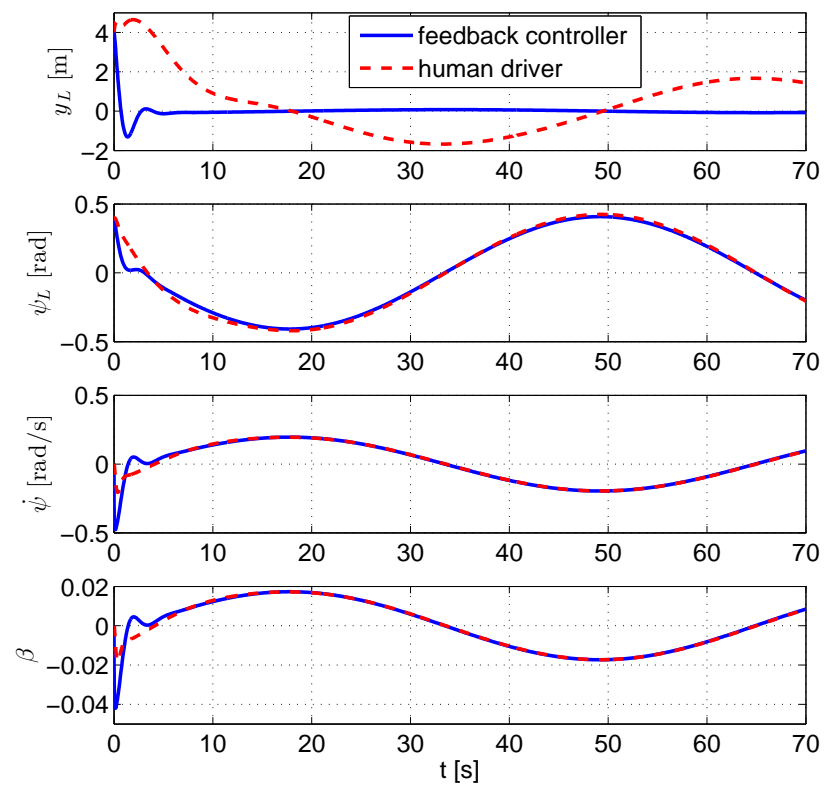

Fig. 8: Tortuous Path Tracking. Time histories of the lateral deviation $y_{L}$, heading error $\psi_{L}$, yaw rate $\dot{\psi}$ and the ratio between the lateral speed and the longitudinal speed $\beta$ for the system (5)-(6)-(7)-(8) with the controller (24) and with a typical human driver.

given feedback controller is much faster than that with the human driver. With the feedback controller (24) the lateral deviation is almost zero within $4 \mathrm{~s}$, while the settling time for the closed-loop system with the human driver is about $20 \mathrm{~s}$. In addition, the maximum lateral deviation is reduced from $3.2 m$ to $0.3 m$ by using the feedback controller.

\section{Tortuous Path Tracking}

In this case study the reference trajectory is a winding and tortuous path, with the curvature of the reference path defined as

$$
\rho(t)=0.02 \sin (0.1 t), \forall t \geq 0
$$

Simulation results are displayed in Fig. 8. Unlike the previous case study, the car with the human driver is unable to track the given reference trajectory. In the case study, we assume that the initial lateral deviation and the initial yaw error are $4 \mathrm{~m}$ and $0.4 \mathrm{rad}$, respectively. With the feedback controller, the closedloop system is able to settle down within $4 s$, while with the human driver, the lateral deviation is nonzero even after $70 \mathrm{~s}$. This demonstrates that the feedback controller (24) is much more effective when the curvature of the reference trajectory is not constant. Even though the differences between the time histories of the variables $\psi_{L}, \dot{\psi}$ and $\beta$ for two closed-loop systems are small, the differences between the time histories of $y_{L}$ are significant because in this case the dynamics of $y_{L}$
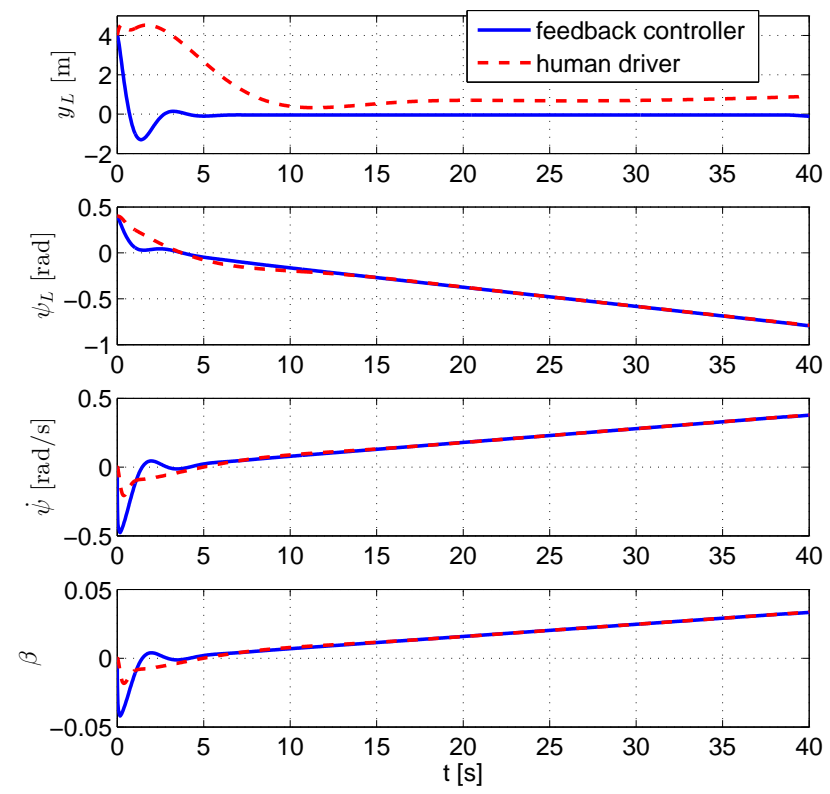

Fig. 9: Spiral Tracking. Time histories of the lateral deviation $y_{L}$, heading error $\psi_{L}$, yaw rate $\dot{\psi}$ and the ratio between the lateral speed and the longitudinal speed $\beta$ for the system (5)(6)-(7)-(8) with the controller (24) and with a typical human driver.

is described by

$$
\dot{y}_{L}=10 \beta+20 \dot{\psi}+10 \psi_{L} .
$$

If we increase the constant longitudinal speed $v_{x}$, then the time histories of the variable $y_{L}$ for two closed-loop systems would have even larger differences.

\section{Spiral Tracking}

This case studies the performance of the system (5)-(6)-(7)-(8) when the curvature of the reference track is defined as

$$
\rho(t)=0.001 t, \forall 0 \leq t \leq 40 .
$$

In other words, the reference path is a spiral the radius of which reduces continuously and uniformly from $+\infty$ to $25 \mathrm{~m}$. We assume that the initial lateral deviation and yaw error are $4 \mathrm{~m}$ and $0.4 \mathrm{rad}$, respectively. Fig. 9 shows the simulation results for this case.

By comparing the settling time for all the three cases, we find out that the settling time for the closed-loop system with the feedback controller is less than $4 s$ no matter what the reference is. In other words, the lateral deviation converges to zero regardless of the variation of the variable $\rho$, which is consistent with Theorem 4. 


\section{CONCLUSIONS}

We have solved the asymptotic stabilization problem for a class of nonlinear systems. The solution, together with backstepping and feed-forward ideas, can be used to develop a lateral controller for autonomous vehicles. We have proved that with the established controller the vehicle is able to track any "feasible" references at a constant speed and the lateral deviation converges to zero within a short period. Even though the control design is based on the lane-keeping case, it can also be applied to lane-changing and other cases by using proper path planning methods to generate a "feasible" reference trajectory that the car follows. In the future, we will devote our efforts to the robust control design of the lateral dynamics of the vehicle in the presence of disturbances and uncertainties. We also aim to implement the controller in a high-fidelity model using CarSim.

\section{REFERENCES}

[1] J. Perez, V. Milanes, and E. Onieva, "Combined longitudinal and lateral control for automated vehicle guidance," Vehicle System Dynamics, vol. 52, no. 2, pp. 261-279, 2014.

[2] S. Li, F. Gao, D. Cao, and K. Li, "Multiple-model switching control of vehicle longitudinal dynamics for platoon-level automation," IEEE Trans. on Vehicular Technology, vol. 65, no. 6, pp. 4480-4492, 2016.

[3] R. Rajamani, Vehicle Dynamics and Control. Springer US, 2012.

[4] H. Zhang and J. Wang, "Vehicle lateral dynamics control through afs/dyc and robust gain-scheduling approach," IEEE Transactions on Vehicular Technology, vol. 65, no. 1, pp. 489-494, 2016.

[5] G. Han, W. Fu, W. Wang, and Z. Wu, "The lateral tracking control for the intelligent vehicle based on adaptive pid neural network," Sensors, vol. 17, no. 6, 2017.

[6] J. Yang and N. Zheng, "An expert fuzzy controller for vehicle lateral control," in Proc. of IEEE Annual Conference on Industrial Electronics, 2007 , pp. 880-885.

[7] X. Huang, H. Zhang, G. Zhang, and J. Wang, "Robust weighted gainscheduling $H_{\infty}$ vehicle lateral motion control with considerations of steering system backlash-type hysteresis," IEEE Transactions on Control Systems Technology, vol. 22, no. 5, pp. 1740-1753, 2014.

[8] C. Latrach, M. Kchaou, A. E. Hajjaji, and A. Rahbi, "Robust $H_{\infty}$ fuzzy networked control for vehicle lateral dynamics," in Proc. of IEEE Annual Conference on Intelligent Transportation Systems, 2013, pp. 905-910.

[9] R. Wang, Y. Sun, M. Lin, and H. Zhang, "Research on bus roll stability control based on LQR," in Proc. of International Conference on Intelligent Transportation, Big Data and Smart City, 2015, pp. 622-625.

[10] S. Lee and C. Chung, "Predictive control with sliding mode for autonomous driving vehicle lateral maneuvering," in Proc. of American Control Conference, 2017, pp. 2998-3003.

[11] G. Tagne, R. Talj, and A. Charara, "Higher-order sliding mode control for lateral dynamics of autonomous vehicles, with experimental validation," in Proc. of IEEE Intelligent Vehicles Symposium, 2013, pp. 678-683.

[12] C. Hatipoglu, U. Ozguner, and K. Redmill, "Automated lane change controller design," IEEE Transactions on Intelligent Transportation Systems, vol. 4, no. 1, pp. 13-22, 2003.

[13] J. Jiang and A. Astolfi, "A lateral control assistant for the dynamic model of vehicles subject to state constraints," in Proc. of IEEE Conference on Decision and Control, Melbourne, Australia, 2017.

[14] B. Gutjahr, L. Gröll, and M. Werling, "Lateral vehicle trajectory optimization using constrained linear time-varying MPC," IEEE Transactions on Intelligent Transportation Systems, vol. 18, no. 6, pp. 1586$1595,2017$.

[15] J. Levinson, J. Askeland, J. Becker, J. Dolson, D. Held, S. Kammel, J. Kolter, D. Langer, O. Pink, V. Pratt, M. Sokolsky, G. Stanek, D. Stavens, A. Teichman, M. Werling, and S. Thrun, "Higher-order sliding mode control for lateral dynamics of autonomous vehicles, with experimental validation," in Proc. of IEEE Intelligent Vehicles Symposium, 2011, pp. 163-168.
[16] P. Falcone, F. Borrelli, J. Asgari, H. Tseng, and D. Hrovat, "Predictive active steering control for autonomous vehicle systems," IEEE Transactions on Control Systems Technology, vol. 15, no. 3, pp. 566-580, 2007.

[17] J. Ahmadi, A. Sedigh, and M. Kabganian, "Adaptive vehicle lateral-plane motion control using optimal tire friction forces with saturation limits consideration," IEEE Transactions on Vehicular Technology, vol. 58, no. 8, pp. 4098-4107, 2009.

[18] J. Ni, J. Hu, and C. Xiang, "Envelope control for four-wheel independently actuated autonomous ground vehicle through AFS/DYC integrated control," IEEE Transactions on Vehicular Technology, vol. PP, no. 99, pp. 1-1, 2017.

[19] S. Dominguez, A. Ali, G. Garcia, and P. Martinet, "Comparison of lateral controllers for autonomous vehicle: Experimental results," in Proc. of IEEE Conference on Intelligent Transportation Systems, 2016, pp. 14181423.

[20] G. Tagne, R. Talj, and A. Charara, "Design and comparison of robust nonlinear controllers for the lateral dynamics of intelligent vehicles," IEEE Transactions on Intelligent Transportation Systems, vol. 17, no. 3 , pp. 796-809, 2016.

[21] G. Kaliora and A. Astolfi, "Nonlinear control of feedforward systems with bounded signals," IEEE Transactions on Automatic Control, vol. 49, no. 11, pp. 1975-1990, 2004.

[22] P. Kokotovic, "The joy of feedback: Nonlinear and adaptive," IEEE Control Systems, vol. 12, no. 3, pp. 7-17, 1992.

[23] C. Sentouh, P. Chevrel, F. Mars, and F. Claveau, "A sensorimotor driver model for steering control," in Proc. of IEEE Conference on Systems, Man and Cybernetics, 2009, pp. 2462-2467.

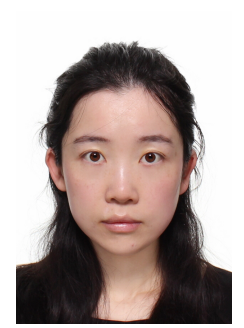

Jingjing Jiang was born in China. She received the B.Eng. Degree in Electrical and Electronic Engineering from the University of Birmingham, UK, and the Harbin Institute of Technology, China, in 2010, the M.Sc. degree in Control Systems with Department Prize for Outstanding Achievement from Imperial College London, UK, in 2011, and the Ph.D degree from Imperial College London, in 2016, with a Thesis on Shared-Control for Systems with Constraints. Since 2016 she has been with the Electrical and Electronic Engineering Department of Imperial College London, London (UK), where she is currently a research associate in the Control and Power Group. Her current research interests include driver assistance control and autonomous vehicle control design, control design of systems with constraints and human-in-the-loop.

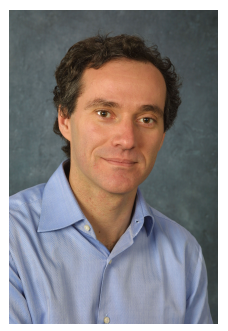

Alessandro Astolfi (F', 2009) was born in Rome, Italy. He received the Bachelor Degree in Electrical Engineering from the University of Rome, Rome, in 1991, the M.Sc. in Information Theory and the Ph.D. (Hons.) with a Thesis on Discontinuous Stabilization of Nonholonomic Systems from ETH Zurich, Zrich, Switzerland, in 1995, and the Ph.D. degree on nonlinear robust control from the University of Rome in 1996. He has been with the Electrical and Electronic Engineering Department, Imperial College London, London, UK., since 1996, where he is currently a Professor of Nonlinear Control Theory and the Head of the Control and Power Group. He was an Associate Professor with Politecnico of Milano, Milan, Italy, from 1998 to 2003. He has also been a Professor at University of Rome Tor Vergata, Rome, since 2005. His current research interests include control theory and applications, with special emphasis for the problems of discontinuous stabilization, robust and adaptive control, observer design and model reduction. Dr. Astolfi, a Distinguished Member of the IEEE Control Systems Society (CSS), was a recipient of the IEEE CSS A. Ruberti Young Researcher Prize in 2007, and the IEEE CSS George S. Axelby Outstanding Paper Award in 2012 